\title{
Late relapse of diabetes after bariatric surgery should not be considered as a failure
}

\begin{abstract}
ABSTRCT
Background: Although the impressive metabolic effects of bariatric surgery in patients with Type 2 Diabetes (T2DM) are known, bariatric surgery is criticized for late relapse of diabetes.

Methods: Outcomes of 736 patients with T2DM who underwent Roux-en-Y Gastric Bypass (RYGB) and Sleeve Gastrectomy (SG) at an academic center (2004-2012) and had $\geq 5$-year glycemic follow-up were assessed. Out of 736 patients, 425 (58\%) experienced diabetes remission (HbA1c $<6.5 \%$ off medications) in the first year after surgery. The latter subgroup was followed to characterize late relapse of T2DM which was defined as fasting glucose (FBG) or $\mathrm{HbA1c}$ in the diabetic range $(\geq 126 \mathrm{mg} / \mathrm{dL}$ and $\geq 6 \cdot 5 \%$, respectively) or need for antidiabetic medication after initial remission.

Findings: The median postoperative follow-up time was 8 years (range, 5- 14). Of those 425 patients who initially achieved remission in shortterm, 136 (32\%) had a late relapse of T2DM. Independent predictors of late relapse were the preoperative number of diabetes medications (OR:1·85,95\%CI:1·35-2.53, $\mathrm{p}=0 \cdot 0001)$, duration of T2DM (OR:1·08,95\%CI:1.02$1 \cdot 15, \mathrm{p}=0 \cdot 012$ ), and SG vs RYGB (OR:1·95,95\%CI:1·00-3·70,p=0·049). In patients who experienced late relapse, a significant improvement in glycemic control, number of diabetes medications including the use of insulin, blood pressure, and lipid profile was still observed at longterm. Among patients with relapse, $77 \%$ maintained glycemic control (HbA1c <7\%).

Interpretation: While late relapse is a real phenomenon (one-third of our cohort), relapse of T2DM years after bariatric surgery should not be considered as a failure, as the trajectory of cardio-metabolic risk factors is changed by surgery. Earlier surgical intervention and RYGB (compared with SG) would be associated with less diabetes relapse in long-term.
\end{abstract}

.Keyword: Bariatric surgery; Gastric bypass; Roux-en-Y gastric bypass; Sleeve gastrectomy; Type 2 diabetes mellitus; Remission, Relapse; Cardiovascular risk. 